\title{
La démarche de développement durable : un processus intégrateur des enjeux sociaux et environnementaux dans les organisations
}

\author{
Olivier Riffon ${ }^{\mathrm{a}}$
}

RÉSUMÉ. Le développement durable est un concept faisant l'objet d’une large adhésion, autant auprès des organisations économiques, sociocommunautaires, environnementales que politiques. Sa définition, proposée en 1987 dans le rapport Brundtland, fait généralement consensus, mais l'interprétation de sa définition tend à varier selon les contextes d'application. De même, les outils utilisés pour sa mise en œuvre varient selon le contexte et les besoins. Au Québec et ailleurs dans le monde, des organisations de toute taille ont entamé des démarches de développement durable. Divers outils, instruments et processus de mise en œuvre sont utilisés pour guider la démarche de ces organisations. Le présent article propose une typologie de ces instruments. Nous présentons également les avantages et limites d’une démarche intégrée de développement durable.

\begin{abstract}
The concept of sustainable development is now widely supported by economic, social, environmental and political organizations. There is a consensus on its definition, proposed in the Brundtland report (1987), but the interpretation of its definition tends to vary according to the context of application. Similarly, the implementation tools use to vary according to context and needs. In Quebec and elsewhere in the world, organizations of all sizes have taken steps towards sustainable development. Various tools, instruments and processes of implementation are used to guide these organizations. This article proposes a typology of these instruments and present the advantages and limitations of an integrated sustainable development process.
\end{abstract}

\section{Introduction}

Depuis son entrée dans le vocabulaire, le développement durable (DD) est devenu un concept faisant l'objet d'une large adhésion, autant auprès des organisations économiques, sociocommunautaires, environnementales que politiques. Sa définition, proposée en 1987 dans le rapport Brundtland, fait généralement consensus. En touchant plusieurs types d'acteurs et d'organisations, le concept a toutefois affiché différents visages et l'interprétation de sa définition tend à varier selon les besoins, la culture et la hiérarchie des valeurs de celui qui s'en approprie l'intention. De même, les outils utilisés pour sa mise en œuvre varient selon le contexte et les besoins.

Au Québec et ailleurs dans le monde, des organisations de toute taille ont entamé des démarches de DD. Divers outils, instruments et processus de mise en œuvre sont utilisés pour guider la démarche de ces organisations. Le présent article propose une typologie de ces instruments. Nous présentons également les avantages d'une démarche intégrée de développement durable. Celle-ci requiert des processus souples qui permettent de faire place aux priorités, de s'adapter aux contextes, besoins et enjeux spécifiques de chaque organisation. Dans ce contexte, les acteurs et groupes d'acteurs impliqués vont construire leur propre représentation du DD et des principes qui lui sont associés, puis identifier les instruments pertinents pour sa mise en œuvre.

\section{Un concept polysémique}

La mise en œuvre d'un développement durable représente un vaste mouvement, autant international que national ou local, qui s'articule autour d'un objectifgénéralement reconnu et énoncé dans le rapport Brundtland : mettre en œuvre « une forme de développement qui doit permettre aux générations actuelles de

\footnotetext{
a Professeur associé, chargé de cours, Université du Québec à Chicoutimi 
répondre à leurs besoins sans compromettre la capacité des générations futures de répondre aux leurs ». Dans sa conception la plus courante, il est compris qu'un développement durable doit être capable d'intégrer simultanément les dimensions écologique, sociale et économique. Le concept obtient l'adhésion d'un large spectre d'acteurs sociopolitiques, de plusieurs agents économiques ou industriels, ainsi que de plusieurs individus et acteurs de la société civile.

En raison de sa définition large et de ses divers champs d'application, une multitude d'interprétations du concept coexiste dans ce paradigme encore en émergence (Gagnon, 2008). Sa constitution en un cadre général dont seuls les principes généraux sont explicités permet une appropriation différenciée par les acteurs, induisant également une diversité d'approches et de stratégies pour son opérationnalisation.

Ainsi, dans le cas d'une organisation, le contenu du concept de DD ainsi que les stratégies et les approches de mise en œuvre restent à définir selon les caractéristiques et la culture organisationnelles, les objectifs fixés et la nature des compétences présentes dans l'organisation. Il est possible d'observer une variance dans la formulation et dans le contenu des plans de développement durable. L'importance accordée aux enjeux écologiques, sociaux et économiques varie, tout comme la nature des processus participatifs et collaboratifs sollicités.

La polysémie du concept est susceptible d'entraîner une certaine confusion auprès des acteurs, avec des conséquences potentiellement néfastes. En l'absence de repères terminologiques et conceptuels, les risques de confusion et d'actions contre-productives sont importants, ce qui représente un défi pour les administrations qui mettent en œuvre des démarches de DD. Ces incompréhensions peuvent mener à des choix d'instruments inappropriés, à des conflits portant sur la nature même du DD, voire à un désengagement des acteurs impliqués.

\section{Des instruments de mise en œuvre du DD}

Plusieurs instruments permettent d'aborder le concept de durabilité de façon opératoire. Dans les dernières décennies, plusieurs outils, instruments et processus d'application, de gestion et de suivi du développement durable ont été développés. Certains sont d'ampleur planétaire alors que d'autres sont plus spécifiques à certains contextes. Plusieurs ont été développés afin de guider les organisations vers un mode de développement plus durable. Ces instruments sont de qualité variable, ils ont des fonctions et des portées diverses. De même, les diverses organisations qui élaborent ou soutiennent ces outils véhiculent des représentations différentes du développement durable.

Les instruments sont des outils qui peuvent être utilisés de façon ponctuelle ou spécifique dans le cadre d'une démarche de développement durable. Ils sont de divers types, et nous les présentons dans le tableau 1, classés selon une typologie inspirée notamment de Lascoumes et Le Galès (2005).

\begin{tabular}{|l|l|}
\hline \multicolumn{1}{|c|}{ Type d'instrument } & \multicolumn{1}{|c|}{ Fonction de l'instrument } \\
\hline $\begin{array}{l}\text { D'orientation et de } \\
\text { planification }\end{array}$ & Outils qui ciblent les priorités et orientent les actions à mettre en œuvre. \\
\hline $\begin{array}{l}\text { Législatifs et } \\
\text { réglementaires }\end{array}$ & $\begin{array}{l}\text { Outils contraignants sous la responsabilité d'une autorité réglementaire } \\
\text { (gouvernement national ou local), d'application obligatoire. }\end{array}$ \\
\hline $\begin{array}{l}\text { Économiques et } \\
\text { fiscaux }\end{array}$ & $\begin{array}{l}\text { Outils qui utilisent des mécanismes de marché, de manière incitative ou } \\
\text { punitive. }\end{array}$ \\
\hline $\begin{array}{l}\text { Informationnels et } \\
\text { de communication }\end{array}$ & $\begin{array}{l}\text { Outils qui permettent d'informer, de sensibiliser, de former, de mobiliser } \\
\text { les parties prenantes avec une visée participative. }\end{array}$ \\
\hline $\begin{array}{l}\text { Normatifs } \\
\text { Technologiques }\end{array}$ & $\begin{array}{l}\text { Outils de référence développés par des autorités compétentes, mais } \\
\text { contribuer au développement durable. }\end{array}$ \\
\hline $\begin{array}{l}\text { D'analyse, de } \\
\text { mesure, de suivi }\end{array}$ & $\begin{array}{l}\text { Outils qui permettent d'évaluer la performance des politiques, stratégies, } \\
\text { programmes et projets, et pour la reddition de compte. }\end{array}$ \\
\hline $\begin{array}{l}\text { Démarches } \\
\text { Processus de planification et d'intervention qui mobilisent différents outils } \\
\text { de développement durable. }\end{array}$ \\
\hline
\end{tabular}

Tableau 1 - Typologie des instruments de mise en œuvre du développement durable 
Plusieurs de ces instruments peuvent être utiles lors de la mise en œuvre de projets de développement durable. Mais si les instruments utilisés par une organisation doivent lui permettre d'agir en concordance avec les principes et objectifs du développement durable, ils doivent être également concordants avec la culture organisationnelle, c'est-à-dire qu'ils doivent être cohérents avec les contextes organisationnel et socioculturel au sein desquels ils seront utilisés. Les divers instruments mobilisés doivent également être concordants entre eux, c'est-à-dire compatibles, articulés et intégrés. La superposition et l'harmonisation des instruments sont des exercices difficiles et complexes à appréhender.

Des instruments mobilisés à la pièce permettent de progresser, mais il demeure difficile de colliger l'information sur l'ensemble des projets et de rendre les apprentissages transférables à d'autres situations. L'absence d'un système de gestion de l'information, tout comme le manque de processus intégrés et transversaux, limite la cohérence de l'ensemble des actions. Ce double objectif de transversalité et de concordance peut être plus facilement atteint par le recours à des démarches de mise en œuvre de développement durable.

\section{Une démarche de développement durable}

Si les organisations ont à leur disposition un grand nombre d'instruments, leur utilisation n'est donc pas seule garante d'un développement plus durable. Un véritable engagement devrait être articulé dans une démarche structurée, lisible, claire et planifiée. C'est pourquoi, au-delà des instruments, les démarches de mise en œuvre du développement durable semblent les plus porteuses de changement pour les organisations (Schneider et Association française de normalisation, 2010).

Nous définissons une démarche de développement durable de la façon suivante : c'est un processus participatif et intégré de planification et d'intervention cherchant à concrétiser la vision du développement durable d'une organisation par la mobilisation de divers instruments. Une démarche est considérée comme un processus continu, qui propose un cadre d'application général pour guider et orienter les modalités de mise en œuvre du développement durable dans une organisation. Elle s'opère à grande échelle, sur l'ensemble de l'organisation et sur le long terme.
Une telle démarche permet de dépasser la simple juxtaposition d'actions et elle se traduit par une approche construite, qui peut véhiculer une représentation ou une vision spécifique du développement durable.

Dans une démarche de développement durable, la dynamique de développement durable est d'abord axée sur le processus, structuré et cohérent, plutôt que sur les résultats prévisibles. Les efforts sont souvent axés sur la qualité et la rigueur de la démarche, qui devraient malgré tout mener à des résultats concrets pouvant être mesurés par des indicateurs de développement durable et faire l'objet d'une reddition de comptes. La démarche est réévaluée sur une base régulière afin de valider que l'organisation évolue dans le sens désiré, soit celui défini par ses objectifs de développement durable.

Ces éléments sont cohérents avec la démarche proposée par le PDCA (Plan Do Check Act), outil de gestion organisationnelle fréquemment utilisé. Nous croyons qu'un cadre de planification stratégique peut être une approche pertinente pour encadrer l'élaboration et la mise en œuvre d'une démarche de développement durable.

\section{Le cadre de la planification stratégique}

La planification stratégique est un exercice de gestion effectué couramment dans divers types d'organisations. Historiquement utilisée dans le cadre de la gestion administrative, la planification stratégique a évolué dans les dernières décennies d'abord pour intégrer les considérations environnementales (la norme ISO 14001), puis pour intégrer les enjeux de développement durable. Le guide BNQ 21000 propose une démarche semblable pour les entreprises et autres organisations.

La planification stratégique de développement durable est une démarche d'amélioration continue flexible, adaptable et qui permet un suivi rigoureux. Les principales étapes d'un exercice de planification stratégique de développement durable ont été maintes fois décrites (Dion et Cadieux, 2012; Ferrand, 2000). Sur la base de différents modèles, nous définissons ces diverses étapes de la façon suivante : l'engagement et la structuration, la planification (bilan, stratégie et plan d'action), la mise en œuvre, la mesure et la reddition de comptes, puis la réévaluation (voir la figure 1). Chacune de ces étapes répond à des objectifs particuliers et peut nécessiter le recours à des outils spécifiques. 


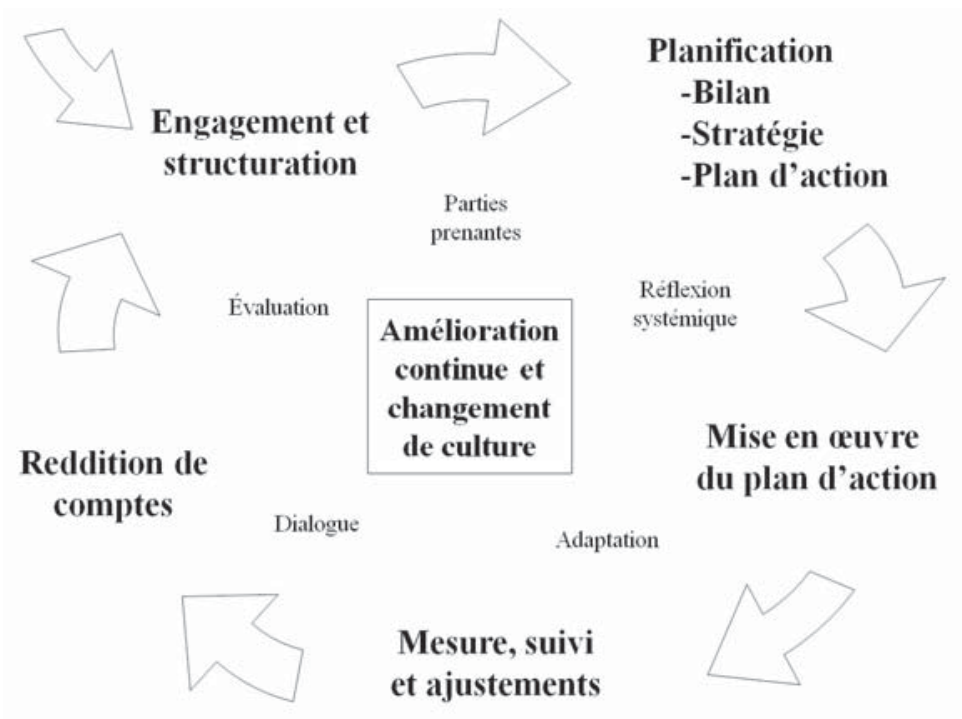

Figure 1-Cycle de planification stratégique du développement durable

\subsection{L'engagement et la structuration}

La première étape du processus est l'engagement, c'est-à-dire l'affirmation de la volonté de l'organisation à accomplir des gestes concrets pour le développement durable. Cette première étape est toutefois le résultat d'un processus de réflexion déjà amorcé en amont de l'engagement. L'engagement doit émaner autant de la direction que des parties prenantes d'une organisation. L'engagement des dirigeants semble toutefois être une condition de l'intégration réelle du DD dans une organisation.

À cette étape, l'organisation va se doter d'une définition commune du développement durable, s'interroger sur les facteurs qui motivent l'engagement de l'organisation, et élaborer la vision et les objectifs organisationnels généraux en matière de développement durable. C'est aussi l'étape où la structure de la démarche est établie. L'organisation doit en définir les principales étapes, les jalons. Elle doit définir les échéanciers, les budgets, et les ressources financières, techniques et humaines qui seront mobilisées. Elle doit identifier les responsabilités et former les comités chargés de la mise en œuvre. Cette étape implique une première mobilisation des parties prenantes, dans une approche participative qui favorise leur implication active aux processus décisionnels.

Au terme de l'étape de l'engagement et de la structuration, une politique de développement durable peut alors faire état du consensus établi dans l'organisation. Cette politique peut préciser la vision, les valeurs, les principes guidant l'organisation dans sa démarche, et en préciser les mécanismes de gouvernance.

\subsection{Planification}

La planification se subdivise en trois sous-étapes : le bilan, la stratégie et le plan d'action. Le bilan est une phase d'acquisition de connaissances qui consiste d'abord à répertorier les actions et les initiatives de développement durable qui sont déjà en place, afin de connaitre le point de départ de l'organisation (portrait ou état des lieux). Cette collecte d'information va permettre d'identifier certains paramètres et indicateurs pertinents à documenter. La réalisation du portrait sert de base au diagnostic organisationnel en matière de développement durable, qui consiste à analyser le portrait réalisé de l'organisation ainsi que son environnement externe pour en faire ressortir des forces et des faiblesses, des opportunités et des contraintes, puis d'identifier les enjeux prioritaires (environnement, social, économique).

L'élaboration de la stratégie de mise en œuvre du développement durable implique souvent la réalisation d'un exercice de prospective et la définition d'une vision future de l'organisation, sur la base de laquelle seront définis les grandes orientations et les objectifs prioritaires. La vision stratégique est une représentation explicite du futur souhaité, une image globale qui indique où l'organisation espère se trouver, en matière 
de développement durable, dans un horizon de planification à long terme, soit dans 15 à 20 ans ou plus. Les stratégies qui sont élaborées doivent permettre de progresser vers cette vision, tout en étant en cohérence avec la politique et le bilan, en considérant les forces et les faiblesses de l'organisation, les opportunités et les contraintes en matière de DD.

Le plan d'action de développement durable est l'outil qui concrétise les objectifs précisés dans la stratégie de développement durable, en proposant une série d'actions pour chaque orientation et objectif identifié. Ce document permet de bien planifier l'ensemble des gestes qui seront mis en œuvre dans une organisation. L'organisation, la hiérarchisation et l'évaluation de la faisabilité des actions sont donc des étapes essentielles qui doivent être effectuées à la suite de l'identification des actions. Le plan d'action devrait également préciser les modalités de mise en œuvre (indicateurs et cibles, responsabilités, budgets, échéanciers), le tout dans une perspective systémique.

\subsection{La mise en œuvre, le suivi et la reddition de comptes}

La mise en œuvre implique la réalisation des actions établies, selon les modalités prévues, et en impliquant l'ensemble des parties prenantes, en accord avec les responsabilités qui ont été précisées. Le succès de cette mise en œuvre repose sur différents facteurs, dont la motivation et la qualité des outils de suivi, mais également sur l'utilisation d'outils de sensibilisation, de conscientisation et de mobilisation des acteurs. La mise en œuvre doit rester flexible et permettre l'adaptation au contexte changeant de l'organisation. D'où l'importance de mécanismes de suivi.

La phase de suivi implique notamment la mesure de l'efficacité des actions mises en œuvre. L'objectif est de vérifier la progression des indicateurs, de vérifier l'atteinte ou non des cibles fixées dans le plan d'action, et de réagir rapidement en mettant en œuvre des mesures de correction ou de bonification. Plusieurs instruments permettent d'évaluer la progression des actions de développement durable d'une organisation : systèmes d'indicateurs, grilles d'analyse, systèmes de gestion de l'information, vérifications internes ou externes (audits). La plus grande transparence est essentielle lors de la mesure et de l'analyse de la performance, puisqu'il vaut mieux conclure qu'une action n'a pas fonctionné et en trouver les raisons que de tenter de se faire croire que les efforts consentis ont porté ses fruits si cela n'est pas vrai.

Grâce au suivi, l'organisation va éventuellement pouvoir rendre compte de ses actions, de ses défis et de ses succès. La reddition de comptes permet de répondre à un objectif de transparence et de publiciser la démarche auprès de différents publics, notamment des publics internes à l'organisation (employés, dirigeants, actionnaires) et des publics externes (clients, gouvernements, organismes subventionnaires). La reddition de comptes peut être faite par des rapports de développement durable ou des rapports annuels intégrés, des bilans de mise en œuvre du plan d'action, des communiqués, des capsules, des campagnes de promotion, etc.

Les étapes de mise en œuvre, de suivi et de reddition de comptes impliquent un dialogue constant avec les parties prenantes, auprès desquelles il importe de partager les informations, les succès, les retombées positives et les obstacles rencontrés.

\subsection{L'évaluation}

Un système de gestion du développement durable vise l'amélioration continue d'une organisation par un processus cyclique, d'où l'importance d'un processus d'évaluation impliquant un retour sur l'expérience et une réflexion sur la poursuite de la démarche. L'évaluation considère la démarche en elle-même, la nature des actions, projets ou politiques proposés, la capacité de l'organisation à atteindre ses objectifs, la progression générale de la démarche et l'impact général en matière de développement durable.

Ces étapes représentent un modèle général et non une méthode applicable dans toutes les situations. Certaines étapes peuvent être regroupées ou esquivées. Une organisation peut aussi faire des allers-retours entre ces étapes. Les processus de planification sont organiques et peuvent être désordonnés, et malgré la volonté de mettre en œuvre une démarche globale et ordonnée, il peut être nécessaire de réaliser des mesures immédiates et d'adapter les actions à l'évolution du contexte (Seymoar, 2008; Vergnet-Covo, 2007).

Retenons que différents instruments seront mobilisés dans une démarche de développement durable. En nous référant au tableau 1 , nous constatons qu'une telle démarche de planification stratégique du développement durable mobilise des instruments de pla- 
nification et d'orientation, des instruments de mobilisation et de communication, des instruments de suivi et de mesure. Les stratégies et actions qui seront entreprises peuvent mobiliser des instruments réglementaires, économiques et technologiques, mais aussi d'autres instruments qui permettent d'intégrer les principes de développement durable dans les modalités de gouvernance, tels les processus d'aide à la décision et les outils d'analyse multicritères. En ce sens, une démarche de développement durable devrait faciliter l'intégration efficace et cohérente des divers instruments du développement durable mobilisés par une organisation.

\section{Vers une démarche intégrée}

Les organisations sont souvent prises dans une organisation sectorielle qui entre en contradiction avec la transversalité qui est souhaitée dans une démarche de développement durable. Une plus grande transversalité qui vise à éviter le travail en silo de compétence serait même une condition de l'efficacité des projets de développement durable. Une démarche de planification stratégique vise à favoriser une gestion plus intégrée des enjeux de développement durable, par l'opportunité qu'elle offre d'effectuer une réflexion globale et transversale concordante avec la complexité des enjeux de développement durable. L'intégration du développement durable devrait se faire avec une vision élargie, comprenant l'atteinte de buts écologiques, sociaux, économiques, culturels, politiques et éthiques déterminés collectivement. C'est une démarche globale, appliquée à très large échelle, dans l'ensemble des secteurs, des départements ou des territoires couverts par une organisation.

Le développement durable étant appliqué dans une perspective globale, intégrée et transversale, une telle démarche va souvent être sous la responsabilité de la direction générale, qui s'assurera d'en intégrer les principes directement dans les processus de prise de décision. En sollicitant la création de comités multiacteurs, la démarche peut contribuer à créer des passerelles entre les silos sectoriels, et favoriser une plus grande transversalité dans les organisations.

Cependant, il n'est pas évident pour une organisation qui apprivoise le concept du développement durable d'intégrer ces notions complexes qui devront un jour modifier sa gouvernance. Il est souvent possible d'observer dans les organisations une évolution du degré d'intégration du développement durable à la stratégie globale de l'organisation, passant d'une démarche parallèle à une démarche intégrée. La première implique la dissociation de la sphère de gestion (administrative ou financière) des activités sociales ou environnementales. Dans une démarche parallèle, le développement durable est abordé par une démarche spécifique, complémentaire aux autres actions, mécanismes et processus de gestion de l'organisation : les préoccupations en matière de développement durable, et elles sont plutôt gérées à part comme quelque chose d'additionnel. La démarche est menée par un comité distinct, la responsabilité étant confiée à des divisions particulières spécialisées dans la durabilité ou la responsabilité sociale. Les discussions et les décisions concernant le développement durable se déroulent alors à l'extérieur des divisions opérationnelles, ou du moins, leur intégration aux opérations se fait dans un second temps.

Dans une démarche parallèle, les problématiques liées au développement durable, considérées comme extrafinancières, impactent peu les décisions stratégiques. Il est aussi possible de voir dans ces processus un risque de dédoublement des processus de planification déjà existants, ce qui entraîne le risque que la démarche de développement durable entre en contradiction avec d'autres politiques ou décisions opérationnelles de l'organisation.

Si une démarche parallèle a moins de chance de faire évoluer la culture de l'organisation vers une prise en compte plus grande des principes du développement durable, elle est souvent, pour une organisation, un passage vers une démarche intégrée, ou les principes de développement durable sont systématiquement intégrés dans les actions, outils et processus de gestion de l'organisation, où l'intégration des critères extrafinanciers gagne le système de gestion de l'organisation, ce que nous appelons la démarche intégrée.

À ce stade, on constate une considération du développement durable dans toutes les décisions, dans toutes les actions, chez tous les individus impliqués, ainsi qu'une application systématique des pratiques exemplaires concernant les enjeux de développement durable qui affectent l'organisation. La démarche intégrée est habituellement porteuse de résultats à plus long terme, parce qu'elle vise explicitement la transformation du système de gestion, pour que le développement durable fasse partie intégrante de la façon de vivre au travail, et faire des affaires ou de gérer ses relations avec les parties prenantes. Une démarche 
intégrée peut permettre à une organisation de se positionner avantageusement par rapport à ses parties prenantes comme étant plus responsable, plus innovatrice. Elle permet enfin d'assurer un meilleur arrimage entre les différentes politiques et actions de l'organisation (Dion et Cadieux, 2012; Mathieu et Soparnot, 2008; Sloan, 2009).

Une démarche intégrée implique toutefois que la direction consacre du temps à cette question et que ces principes soient considérés non seulement dans les décisions stratégiques, mais également dans l'allocation des ressources, dans la gestion des ressources humaines, dans l'approvisionnement et dans le marketing (Sloan, 2009). C'est pourquoi la mise en œuvre d'une démarche intégrée représente souvent une seconde étape dans l'intégration des principes du développement durable. Certaines organisations ressentent ainsi le besoin de débuter par une démarche parallèle, afin de mieux circonscrire les limites et la portée du concept du développement durable. Elles cheminent par la suite vers une démarche plus intégrée, lorsque les acteurs ont apprivoisé le concept.

\section{Amélioration continue et culture organisationnelle}

Cette trajectoire d'une démarche parallèle à une démarche intégrée répond bien à l'objectif du développement durable de transformer les modalités de gouvernance des organisations, afin que ce concept devienne un fil conducteur, un guide pour les processus décisionnels. Le développement durable progresse de façon évolutive, dans une perspective d'amélioration continue, de façon ouverte et adaptative. Cette stratégie progressive est à la base de la loi québécoise sur le développement durable, mais aussi du processus de gestion du développement durable proposé par la norme BNQ 21000. Cette stratégie est basée sur l'apprentissage progressif et permet aux organisations d'opérer à leur rythme les changements nécessaires dans leurs pratiques de gestion.

Mathieu et Soparnot (2008) distinguent à cet effet trois types de comportements dans les organisations : 1) les attentistes ou défensifs, qui font diversion ou qui refusent de prendre en compte les responsabilités sociales et environnementales liées au développement durable; 2) les adaptatifs ou les réactifs, qui agissent lorsque forcés par le contexte et; 3 ) les proactifs qui agissent de façon anticipée et font preuve de leadership. Cadieux et Dion (2012) mentionnent pour leur part qu'une organisation peut traverser cinq stades de maturité dans l'attention portée au développement durable : 1) peu ou pas concernée (culture d'insouciance); 2) réactive (culture de résultats); 3) accommodante (culture de conformité ou de contrôle); 4) proactive (culture d'amélioration continue) et; 5) génératrice (culture intégrée). Dans les deux cas, les auteurs considèrent qu'une organisation peut cheminer, évoluer (ou régresser) entre ces stades, ce qui peut être atteint par la mise en place d'une démarche de développement durable. Une telle démarche peut augmenter le niveau de proactivité dont l'organisation fait preuve, passant d'une attitude réactive à une attitude proactive.

Une organisation réactive agit en matière de démarche de développement durable en réaction à une situation critique, à une menace, à une obligation, à des pressions ou à des changements organisationnels importants. Elle respecte les normes, sans aller audelà, tentant ainsi de limiter les risques d'infraction, de minimiser les risques et les oppositions, de minimiser les investissements, tout en visant le maintien du profit et en préservant la légitimité et son image de ses activités. Elle continue de privilégier les actions ayant des rendements immédiats, considérant les investissements en développement durable comme des coûts. La culture est alors dictée par une logique de résultats à court terme, ou encore de rattrapage.

Les organisations peuvent alors reconnaitre l'existence de plusieurs problèmes et peuvent être critiques à l'égard des modes dominants de développement, mais elles ne craignent pas l'effondrement des systèmes écologiques ou sociaux et n'éprouvent pas le besoin pour des changements en profondeur. Parce qu'elles ne situent pas la source des problèmes dans la nature actuelle de la société, elles ne remettent pas en question les bases sur lesquelles la société s'est construite. Ces organisations mettent alors l'accent sur des réformes légères des systèmes socio-économiques pour les rendre, d'un point de vue environnemental, plus responsables et socialement plus justes, en œuvrant à l'intérieur des structures économiques et sociales actuelles et en se concentrant sur des changements marginaux.

Au fil de son évolution, l'organisation va commencer à mettre en place des actions pour le développement durable sans subir de pression, ni interne ni externe. L'internalisation partielle ou totale des enjeux sociaux et environnementaux dans l'organisation se fait de manière 
anticipée et l'organisation s'engage de manière à devancer les problèmes ou les obstacles. Cet engagement se traduit dans les discours, mais également dans les décisions et dans les actes. Une attitude proactive permet de dépasser les exigences réglementaires, puisque l'organisation se donne une obligation de contribuer à gérer les problèmes sociaux. L'organisation considère son engagement envers le développement durable comme un élément clé de sa pérennité, engagement qui apporte des bénéfices sur les plans de la légitimité, de l'image et de la distinction. Cela se produit lorsque les décideurs au sein de l'organisation en arrivent à intérioriser les valeurs qui caractérisent le développement durable. Cette évolution de la culture organisationnelle, d'une attitude réactive qui considère les enjeux du développement durable comme des externalités vers une attitude proactive qui intègre de manière systématique les responsabilités sociales et environnementales de l'organisation, peut être facilitée par la mise en œuvre d'une démarche de plus en plus intégrée de développement durable.

\section{Conclusion}

La mise en œuvre d'une démarche intégrée de développement durable favorise une progression flexible et adaptative des pratiques de l'organisation, orientée vers l'amélioration continue avec une vision claire des objectifs à atteindre. Une telle démarche permet de s'ajuster, en continu, aux variations des conditions du milieu et de l'environnement externe, sans tenter de tout contrôler. Le recours à une démarche permet une adaptation progressive des instruments et des principes du développement durable à la culture de l'organisation, grâce à la réflexion stratégique qui entoure le processus. Elle permet de mobiliser de manière cohérente différents instruments de développement durable, en dépassant la simple juxtaposition d'actions, pour se traduire dans une démarche construite, qui peut véhiculer une représentation claire et une vision spécifique du développement durable par l'organisation.

Une telle démarche peut sembler complexe, être longue à mettre en place et ne pas donner de résultats concrets à court terme, ce qui peut décourager certains individus ou certaines organisations. Elle permet toutefois, à terme, de faire évoluer la culture organisationnelle vers une prise en compte plus systématique et systémique des principes et des enjeux sociaux, économiques, politiques, culturels, écologiques et éthiques du développement durable dans les processus de gestion de l'organisation.

\section{RÉFÉRENCES}

Dion, M. et Cadieux, J. (2012). Manuel de gestion du développement durable en entreprise : une approche progressive : en appui à la norme BNQ 21000. Anjou, Québec : Fides.

Ferrand, D. (2000). Piloter l'environnement dans l'entreprise. Montréal, Québec : Ordre des ingénieurs du Québec éd.

Gagnon, C. (2008). Le développement durable : un nouveau paradigme scientifique? Dans G. Massicotte (dir.), Sciences $d u$ territoire, perspectives québécoises (p. 335-368). Québec, Québec : Presses de l’Université du Québec.

Hopwood, B., Mellor, M. et O’Brien, G. (2005). Sustainable development: mapping different approaches. Sustainable Development, 13, 38-52.

Lascoumes, P. et Galès, P. L. (2005). Gowverner par les instruments. Paris, France : Presses de Sciences Po.

Mathieu, A. et Soparnot, R. (2008). Les stratégies de développement durable. Dans D. Wolff (dir.), Le développement durable. Théories et applications au management. Malakoff, France : Dunod.

Schneider, L. et Association française de normalisation. (2010). Le développement durable territorial. La Plaine Saint-Denis, France : AFNOR Éditions.

Seymoar, N.-K. (2008). Sustainable cities. Communication présentée à la Durban Biennial Conference.

Sloan, P. (2009). L'engagement des dirigeants envers les parties prenantes : condition de succès du développement durable. Gestion, 34(1), 79-88.

Vergnet-Covo, M. (2007). Ville durable : mode d'emploi. Voiron, France : Territorial. 